\title{
ORIGINAL
}

\section{EL COSTE DE MORTALIDAD ASOCIADO AL CONSUMO DE TABACO EN ESPAÑA (*)}

\section{Ma Belén Cobacho Tornel (1), Ángel López Nicolás (2,3) y José María Ramos Parreño (2)}

(1) Departamento de Métodos Cuantitativos e Informáticos. Universidad Politécnica de Cartagena.

(2) Departamento de Economía. Universidad Politécnica de Cartagena.

(3) Centre de Reserca en Economia i Salut - CRES. Universitat Pompeu Fabra.

\section{RESUMEN}

Fundamento: Las políticas públicas juegan un papel crucial en la prevención del tabaquismo y la mejora de la salud de la población. A pesar del impacto positivo de la entrada en vigor de la ley de medidas sanitarias frente al tabaquismo en España en 2006, aún es posible seguir progresando en las medidas de control sobre el consumo de tabaco. La estimación del coste de la mortalidad prematura por paquete de tabaco es un elemento clave en el análisis coste-beneficio de las políticas de prevención y control del tabaquismo. Los objetivos de este estudio son estimar el Valor Estadístico de la Vida (VEV) en la población de fumadores españoles, así como el coste de mortalidad asociado al consumo de tabaco.

Métodos: Utilizamos un modelo de salarios hedónicos que permite cuantificar la valoración marginal de un incremento del riesgo de muerte en términos monetarios. Estimamos dicho modelo para el mercado laboral español, con datos del Panel de Hogares de la Unión Europea y la Encuesta de Accidentes de Trabajo del Ministerio de Trabajo e Inmigración.

Resultados: Estimamos que el VEV en la población de fumadores españoles asciende a 3,78 millones de euros. Utilizando este valor, junto con el incremento en el riesgo mortal a lo largo del ciclo vital causado por el consumo de tabaco, calculamos que el coste de mortalidad asociado al tabaquismo es de 78 euros por paquete de cigarrillos en el caso de los hombres, y 54 euros para las mujeres (euros año 2000).

Conclusiones: El coste por mortalidad prematura por paquete de cigarrillos que se obtiene sobrepasa con creces su precio de venta al público.

Palabras clave: Tabaquismo. Mortalidad. Valor de la Vida.
ABSTRACT

\section{Mortality Cost of Smoking in Spain}

Background: Public policies are crucial for smoking prevention and improving health among the population. Despite the positive impact in Spain of the law for smoking prevention in 2006, there is room for further improvement in this area of public policy. The estimate of the mortality cost per pack of cigarretes is a crucial factor in cost-benefit analysis for policies aimed to reducing smoking induced mortality. The aim of this paper is twofold. First, we estimate the Value of Statistical Life (VSL) among Spanish smokers. Secondly, we quantify the mortality cost of smoking.

Methods: We use a hedonic wage model to quantify the marginal value of an increase in the mortality risk in monetary terms. We estimate the model for the Spanish labour market using the European Community Household Data and the Encuesta de Accidentes de Trabajo from the Ministerio de Trabajo e Inmigración.

Results: We estimate a VSL of 3.78 million Euros for Spanish smokers. Using this value, in conjunction with the increase in the mortality risk over the life cycle due to smoking, the private mortality cost of smoking is 78 Euros per pack for men, and 54 Euros per pack for women (in 2000 Euros).

Conclusions: The mortality cost per pack of cigarettes is highly above its market price.

Key words: Smoking. Economic value of life. Model, Econometric.
Correspondencia:

Ángel López Nicolás

Facultad de Ciencias de la Empresa,

C/ Real, 3

30201 Cartagena

angel.lopez@upct.es
(*) Este artículo es resultado del proyecto de investigación 08646/PHCS/08 financiado por el Programa de Generación de Conocimiento Científico de Excelencia de la Fundación Séneca-ACTRM en el marco del II PCTRM 2007-10, y del proyecto ECO2008-06395-C05-04, del Plan Nacional de I+D (cofinanciado por FEDER). 


\section{INTRODUCCIÓN}

Los economistas de la salud consideran que fumar genera costes y beneficios, tanto a los propios fumadores como al resto de la sociedad. El debate sobre la justificación económica de las políticas de prevención del tabaquismo se ha basado con demasiada frecuencia en los costes externos: perjuicios para los fumadores pasivos, externalidades fiscales, daños materiales y humanos causados por incendios, costes de limpieza de espacios públicos, etc. Sin embargo, siendo de una cuantía abrumadoramente superior a los costes externos ${ }^{1}$, los costes que recaen sobre el propio fumador han recibido menos atención. Posiblemente ello se deba a la tradicional idea de que el fumador los soporta voluntariamente, ya que el beneficio que deriva de fumar es mayor, en ejercicio de una supuesta soberanía del consumidor. Avances recientes en la investigación económica del comportamiento de los fumadores ${ }^{2-}$ ${ }^{4}$ ponen en cuestión el mencionado enfoque tradicional. En concreto, plantean la hipótesis de que los fumadores son conscientes de que el coste de su hábito supera al placer de fumar y, no obstante, no son capaces de abandonarlo. Esta hipótesis es consistente con la evidencia empírica sobre deseos de abandono, intentos fallidos de abandono, y valoración de políticas que ayudan al abandono $^{5-7}$.

En la medida en que el coste privado de fumar no sea plenamente «internalizado» por los fumadores, las evaluaciones de las políticas de prevención del tabaquismo han de incorporar no sólo estimaciones de los costes externos sino también de los costes soportados por el propio fumador. Con este trabajo pretendemos aportar evidencia sobre uno de los elementos, quizás el más importante, que componen dichos costes: el valor de los años de vida perdidos a causa del consumo de tabaco. Para ello utilizamos el concepto de Valor Estadístico de la Vida (VEV), que puede entenderse como la cantidad que una sociedad estaría dispuesta a pagar para reducir riesgos hasta el punto de conseguir una reducción de una muerte en la tasa de mortalidad.

En la literatura reciente se ha utilizado el VEV para estimar el coste de la mortalidad asociada al tabaquismo en Estados Unidos, que se encuentra entre los 20 y los $30 \$$ por paquete de cigarrillos ${ }^{1,4,8}$. La metodología en estos trabajos supone que la pérdida de vida debida al tabaquismo ocurre al final de la trayectoria vital del fumador, y se puede estimar en unos 100.000\$ anuales, basándose en el Valor Medio Anual de la Vida Estadística $(\mathrm{VAEV})^{9}$.

Consideramos que para obtener estimaciones del coste por mortalidad prematura soportado por los fumadores es necesario utilizar estimaciones del VEV en la población de fumadores. Con ello se contempla la posibilidad de que la actitud ante el riesgo, e implícitamente la valoración de la propia vida, difiera de la del resto de la población. No en vano una de las teorías económicas que justifican la decisión de fumar como un ejercicio de plena soberanía del consumidor alega que los fumadores valoran menos la vida en buena salud que el resto de la población en término medio. Un estudio reciente para EEUU ${ }^{10}$ adopta este enfoque: estima el VEV por estatus de fumador, edad y sexo, y considera como coste de mortalidad asociado al tabaquismo el valor monetario presente del incremento en la probabilidad de muerte en diferentes edades que los fumadores soportan, en relación a los no fumadores de las mismas características.

Para el caso español, algunos trabajos han utilizado el método de los salarios hedónicos para estimar el VEV, con resultados entre 2 y 3 millones de euros ${ }^{11,12}$, mientras que otros proponen un método de identificación de cotas para el VEV, obteniendo una cota inferior de 2,8 y una cota superior de 8,3 millones de euros ${ }^{13}$. No obstante, como hemos apuntado, la aplicación de las estimaciones del VEV en la población general podría ses- 
gar al alza las estimaciones de coste desde el punto de vista de los fumadores.

Así pues, con el presente trabajo obtenemos estimaciones del VEV para el caso español diferenciando por estatus de fumador y, a partir de dichas estimaciones, estimamos a su vez el coste privado de mortalidad asociado al tabaquismo. Hasta donde conocemos, ningún estudio anterior se basa en el intercambio entre salario y riesgo para la estimación del coste por mortalidad prematura soportado por los consumidores de tabaco en España.

\section{MATERIAL Y MÉTODOS}

Fuentes de datos: La base de datos utilizada se ha construido a partir de la información que proporciona el Panel de Hogares de la Unión Europea (PHOGUE ${ }^{14}$ ), coordinada por EUROSTAT y elaborada en España por el INE, y la Estadística de Accidentes de Trabajo (EAT) del Ministerio de Trabajo e Inmigración $^{15}$ para el año 2000 , ya que en dicho año se llevó a cabo una ampliación de la muestra del PHOGUE que permite realizar estudios transversales con mayor precisión.

La variable dependiente en los modelos econométricos que estimamos es el logaritmo neperiano del salario anual neto. Se han seleccionado en el PHOGUE aquellos trabajadores asalariados que declaran percibir ingresos por un trabajo con una dedicación semanal superior a las 15 horas. El PHOGUE también suministra información sobre las variables demográficas (situación familiar, formación, vida laboral y experiencia, situación geográfica y estado de salud) y variables relacionadas con el puesto de trabajo (ocupación desempeñada, tamaño de la empresa, nivel de responsabilidad asumido por el trabajador, situación laboral y tipo de jornada, grado de satisfacción, existencia de compensaciones no monetarias del salario, tales como las aportaciones de la empresa en concepto de conservación de la salud y/o mejora de la formación) que pueden determinar el salario. La información sobre el consumo de tabaco ha sido extraída también del PHOGUE, (fumador/ no fumador, y en caso afirmativo, número de cigarrillos consumidos al día). Las variables utilizadas aparecen en la tabla de resultados de las regresiones (tabla 1). Una tabla descriptiva de las mismas está disponible mediante solicitud a los autores.

Índices de riesgo: Se han utilizado en la literatura medidas específicas de riesgo, bien por ocupaciones sin tener en cuenta la variación del riesgo por ramas de actividad ${ }^{11}$, o bien por rama de actividad dejando a un lado la variación del riesgo por ocupación ${ }^{16}$, aunque se recomienda la construcción de índices de riesgo por ocupación y actividad conjuntamente ${ }^{12}$. La EAT suministra el número de accidentes de trabajo mortales y no mortales utilizando las clasificaciones CNO-94 (dos dígitos) para las ocupaciones y CNAE93 para ramas de actividad. En nuestro estudio hemos restringido el número de ocupaciones a nueve, con la finalidad de poder desagregar además por género, tramos de edad o estatus de fumador.

Así pues, el índice de riesgo se calcula, para cada celda de ocupación y actividad, como el número de muertes en esa ocupación y rama de actividad, dividido entre el número de trabajadores existentes en esa ocupación y rama de actividad en España, obtenido a través de los correspondientes factores de expansión que proporciona el PHOGUE. Análogamente se calculan los índices de riesgo no mortal. El índice de riesgo mortal para 9 ocupaciones y 18 ramas de actividad puede encontrarse en el anexo 1 .

El modelo de salarios hedónicos: Este método parte del supuesto de que un trabajador, ante dos puestos de trabajo con características iguales salvo en el riesgo de accidente, sólo aceptará el empleo de mayor riesgo si existe una compensación en térmi- 
nos de salario. De esta manera, los individuos toman decisiones que implican de manera implícita una relación de intercambio entre riesgo y salario. Siguiendo la literatura, utilizamos una forma funcional semilogarítmica para la especificación:

$$
\ln \left(w_{i}\right)=\alpha+X_{i}^{\prime} \beta_{x}+\beta_{m} R_{m}+\beta_{n m} R_{n m}+\varepsilon_{i}
$$

donde $w_{i}$ es el salario del individuo $i, \alpha$ es el término constante, $X_{i}$ es un vector de características personales y variables de control del trabajo asociadas a cada individuo, $\beta_{x}$ es el vector de parámetros de las variables incluidas en $X_{i}, R_{m}$ y $R_{n m}$ son los índices de riesgo mortal y no mortal incorporados al modelo, con coeficientes $\beta_{m}$ y $\beta_{n m}$ respectivamente, y $\varepsilon_{i}$ es el término de error aleatorio. Para corregir el sesgo de selección que puede aparecer en la estimación, utilizamos el método en dos etapas de Heckman ${ }^{17}$. Se han considerado en el probit dos variables (estado civil «casado»y «soltero») que no se incluyen en la ecuación de salarios; de no tener en cuenta esto, el modelo podría no estar bien identificado ${ }^{18}$.

El Valor Estadístico de la Vida: Se calcula a partir del intercambio riesgo mortalsalario revelado por la decisión de los trabajadores acerca de qué cantidad marginal de su salario se requeriría para hacerles aceptar un incremento marginal en el riesgo mortal asociado a su trabajo. Teniendo en cuenta que los índices de riesgo los calculamos en tantos por mil, el VEV medio para el conjunto de la muestra sería $V E V=\frac{\partial w_{i}}{\partial R_{m}} \cdot 1000$. Ahora bien, si derivamos en la ecuación del modelo con respecto al índice de riesgo, se tiene que $\frac{\partial \ln \left(w_{i}\right)}{\partial R_{m}}=\beta_{m}$. Es decir, $\frac{1}{w_{i}} \cdot \frac{\partial w_{i}}{\partial R_{m}}=\beta_{m}$, o equivalentemente, $\frac{\partial w_{i}}{\partial R_{m}}=w_{i} \cdot \beta_{m}$, de donde:

$$
V \hat{E} V=\bar{w} \cdot \hat{\beta_{m}} \cdot 1000
$$

En nuestro estudio calculamos este valor para el conjunto de la muestra en primer lugar, y para fumadores y no fumadores posteriormente.

Coste de mortalidad: El coste $c$ de la mortalidad asociada al tabaquismo se puede calcular como el número esperado de años de vida perdidos debido al hecho de fumar, multiplicado por el valor económico de esos años (el VEV) ${ }^{10}$ :

$$
c=\sum_{t=t_{0}}^{100} \frac{\left(x_{s t}-x_{n t}\right) V E V(t)}{(1+r)^{t-t_{0}}}
$$

donde $t_{0}$ es la edad a la que la persona se convirtió en fumador habitual, $x_{s t}$ es la probabilidad de que este fumador muera a la edad $t$, $x_{n t}$ es la probabilidad de que un no fumador con sus mismas características hubiera muerto a la edad $t, \operatorname{VEV}(t)$ se utiliza como medida del valor (coste) de la muerte a la edad $t$, y $r$ es la tasa de descuento. Este enfoque utiliza como base el perfil de riesgo de un no fumador con el mismo perfil demográfico y de riesgo de un fumador, lo que refleja correctamente el incremento en el riesgo mortal que será experimentado por un fumador debido exclusivamente al hábito de fumar ${ }^{1}$. Tomamos como referencia $t_{0}=24$ años ${ }^{10}$. A esa edad, se supone que los fumadores han adquirido el hábito de fumar normalmente. Para fumadores de 65 años o más no se puede contar con un VEV basado en los intercambios del mercado laboral, de modo que para ese tramo de edad se calcula el VAEV (Valor Medio Anual de la Vida Estadística) a partir del VEV de los trabajadores en el tramo de edad anterior (55-64 años $)^{10}$.

Para el cálculo del coste de mortalidad por paquete de cigarrillos se necesita el valor descontado del número de paquetes fumado $(d)$. Para ello utilizamos la información contenida en el PHOGUE, calculando el número medio de paquetes de cigarrillos fumados por edad y sexo $\left(z_{s t}\right)$. De 
esta forma, $\quad d=\sum_{t=24}^{100} \frac{y_{s t} z_{s t}}{(1+r)^{t-24}} \quad$, siendo $y_{s t}$ la probabilidad de supervivencia para un fumador a la edad de $t$ años ${ }^{1}$.

Finalmente, el coste de mortalidad por paquete de cigarrillos es $c / d$.

\section{RESULTADOS}

Estimaciones de los VEV: La muestra escogida para la estimación del modelo de salarios hedónicos está formada por 11.429 observaciones de las 35.550 con las que cuenta la muestra de adultos ampliada del PHOGUE para el año 2000. La tabla 1 recoge los principales resultados de la estimación.

Obtenemos que el coeficiente del índice de riesgo mortal es significativo, mientras que el del riesgo no mortal no lo es. El valor estimado para el efecto del riesgo mortal sobre el salario es 0,201 , con lo que aplicando la expresión (2) se obtiene un VEV de 2,92 millones de euros (tabla 2).

Partiendo del modelo base, introducimos en la ecuación de salarios una variable cualitativa que representa el hecho de ser o no fumador, interactuando con el índice de riesgo. Los resultados en este caso arrojan un coeficiente estimado del índice de riesgo para los fumadores de $\hat{\beta}_{m}^{s}=0,2693$, frente al de los no fumadores de $\hat{\beta}_{m}^{n s}=0,1452$ (tabla 2). Los coeficientes obtenidos nos llevan a VEV para fumadores y no fumadores de 3,78 y 2,14 millones de euros respectivamente. Esta diferencia en el VEV no es habitual en la literatura anterior; sin duda, el hecho de que entre los no fumadores de la muestra con la que trabajamos haya una mayoría de mujeres, con menores salarios y probablemente menores coeficientes sobre el riesgo de índice mortal (así es en las pruebas que hemos realizado, aunque los coeficientes no resultan significativos), contribu- ye a explicar esta diferencia en el VEV de los fumadores a la baja.

Estimación del coste de mortalidad asociado al consumo de tabaco: Considerando una tasa de descuento del 3\% ( $r=0.03)$, obtenemos las estimaciones para el coste de mortalidad por paquete de cigarrillos $(c / d)$ que presentamos en la tabla 3. En euros del año 2000, los valores obtenidos son de 78,9€ para los hombres y de $54,7 €$ para las mujeres, valores que en euros de 2010 serían de $104,14 €$ y $72,20 €$ respectivamente, lo que indica que el coste de fumar sobrepasa con creces el precio de compra de un paquete de cigarrillos.

\section{DISCUSIÓN}

En concordancia con estudios anteriores $^{11,12}$, obtenemos que el coeficiente del índice de riesgo mortal es significativo, mientras que el de riesgo no mortal no lo es. No obstante es necesario incluir en la ecuación este último índice con el fin de que el índice de riesgo mortal no recoja el posible efecto sobre los salarios del resto de accidentes no mortales ${ }^{11}$. El valor estimado para el efecto del riesgo mortal sobre el salario es de 0,201 , similar al de otros estudios ${ }^{11,12}$. Cuando introducimos una variable cualitativa que representa el hecho de ser o no fumador, se obtiene un coeficiente estimado del índice de riesgo para los fumadores de $\hat{\beta}_{m}^{s}=0,2693$, frente al de los no fumadores de $\hat{\beta}_{m}^{n s}=0,1452$, similar en términos cualitativos a los obtenidos para Estados Unidos ${ }^{10}$.

Este trabajo proporciona la primera estimación del VEV por estatus de fumador y del coste privado de mortalidad asociado al tabaquismo en España. Los VEV por estatus de fumador son muy superiores para fumadores frente a los no fumadores, diferencia que se debe a una mayor proporción de mujeres -que presentan un salario medio inferior al de los hombres- entre los no fumadores. Con una tasa de descuento del 
Tabla 1

Resultados de la estimación de la ecuación de salarios

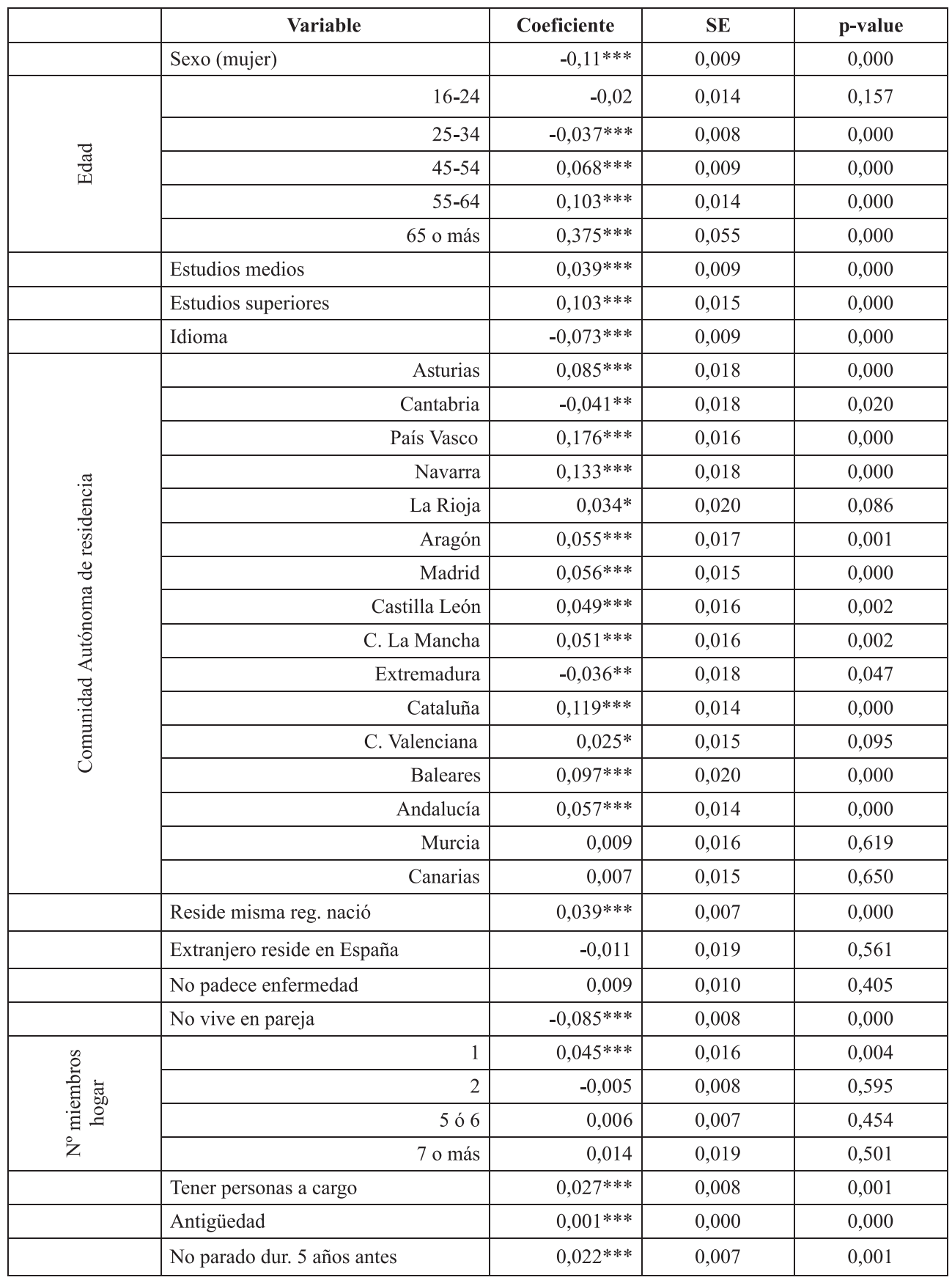


Tabla 1 (Cont.)

Resultados de la estimación de la ecuación de salarios

\begin{tabular}{|c|c|c|c|c|}
\hline & Variable & Coeficiente & SE & p-value \\
\hline \multirow{8}{*}{ 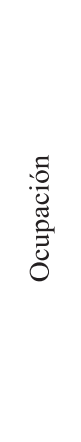 } & Técnicos y profesion. sup. & $-0,179 * * *$ & 0,022 & 0,000 \\
\hline & Técnicos y profesion. apoyo & $-0,366^{* * *}$ & 0,022 & 0,000 \\
\hline & Administrativos & $-0,444 * * *$ & 0,022 & 0,000 \\
\hline & $\begin{array}{l}\text { Trabajadores servicios comerciales y } \\
\text { personales }\end{array}$ & $-0,536^{* * *}$ & 0,022 & 0,000 \\
\hline & Cualificados sector primario & $-0,624 * * *$ & 0,032 & 0,000 \\
\hline & Cualif. industria y construcc. & $-0,504 * * *$ & 0,022 & 0,000 \\
\hline & Operadores y montadores & $-0,518^{* * *}$ & 0,023 & 0,000 \\
\hline & No cualificados & $-0,616^{* * *}$ & 0,023 & 0,000 \\
\hline \multirow{6}{*}{ 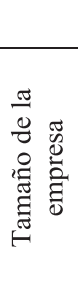 } & Sector público & $0,042 * * *$ & 0,008 & 0,000 \\
\hline & $5-19$ & $0,078 * * *$ & 0,008 & 0,000 \\
\hline & $20-49$ & $0,122^{* * *}$ & 0,009 & 0,000 \\
\hline & $50-99$ & $0,134 * * *$ & 0,011 & 0,000 \\
\hline & $100-499$ & $0,182 * * *$ & 0,010 & 0,000 \\
\hline & 500 o más & $0,2 * * *$ & 0,011 & 0,000 \\
\hline \multirow{14}{*}{ 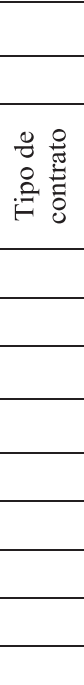 } & No ocupar puesto de respons. & $-0,116$ & 0,007 & 0,000 \\
\hline & Contrato tpo. parcial & $-0,33 * * *$ & 0,017 & 0,000 \\
\hline & Eventual & $-0,069^{* * *}$ & 0,007 & 0,000 \\
\hline & Sin contrato & $-0,258^{* * *}$ & 0,018 & 0,000 \\
\hline & Otro & $-0,076^{* * *}$ & 0,018 & 0,000 \\
\hline & Horas semana $(\log )$ & $0,315^{* * *}$ & 0,019 & 0,000 \\
\hline & Satisfecho con su trabajo & $0,029 * * *$ & 0,006 & 0,000 \\
\hline & Compens. no salariales salud & $-0,029 * * *$ & 0,006 & 0,000 \\
\hline & Compens. no salar. formac. & $-0,05 * * *$ & 0,007 & 0,000 \\
\hline & Índice de riesgo mortal & $0,201 * * *$ & 0,035 & 0,000 \\
\hline & Índice de riesgo no mortal & $-0,001$ & 0,000 & 0,461 \\
\hline & Constante & 13,982 & 0,082 & 0,000 \\
\hline & Lambda & $-0,179 * * *$ & 0,015 & 0,000 \\
\hline & $\mathrm{N}=11.429$ & & & \\
\hline
\end{tabular}

$* * * \mathrm{p}<0.01$ Significativo al $99 \%, * * \mathrm{p}<0.05$ Significativo al $95 \%, * \mathrm{p}<0.1$ Significativo al $90 \%$ 
Tabla 2

VEV para el caso general y por estatus de fumador

\begin{tabular}{|l|r|r|r|}
\hline & General & Fumador & \multicolumn{1}{c|}{ No Fumador } \\
\hline Salarios medios $(*)$ & 2.414 .192 & 2.327 .533 & 2.449 .459 \\
Coef. IRMortal & 0,2007 & 0,2693 & 0,1452 \\
VEV (millones $€$ ) & $\mathbf{2 , 9 2}$ & $\mathbf{3 , 7 8}$ & $\mathbf{2 , 1 4}$ \\
\hline
\end{tabular}

(*) Pesetas año 2000.

Tabla 3

Costes de mortalidad

\begin{tabular}{|l|c|c|c|}
\hline & & Hombre & Mujer \\
\hline Coste mortalidad $(€)$ & $\mathrm{c}$ & 694.108 & 360.954 \\
\hline $\mathrm{N}^{\text {o }}$ paquetes fumados a la edad 24 & $\mathrm{~d}$ & 8794,7 & 6604,4 \\
\hline Coste mortalidad por paquete $(€)$ & $\mathrm{c} / \mathrm{d}$ & 78,9 & 54,7 \\
\hline
\end{tabular}

$3 \%$, el coste de mortalidad por paquete de cigarrillos consumido es de $104,14 €$ para los hombres y de $72,20 €$ para las mujeres (en euros de 2010).

Las cifras que obtenemos para el caso español refrendan la tónica de estudios para otros países, encontrando que el componente por mortalidad prematura -con respecto a un no fumador de características comparables- de los costes privados del tabaquismo supera ampliamente el precio de venta al público. Desde la visión económica ortodoxa del consumo de tabaco (formalizada en la Teoría de la Adicción Racional ${ }^{19}$ ) la conclusión a inferir sería que al consumir cigarrillos, los fumadores revelan que su valoración de un paquete de tabaco supera el coste privado total: el precio pagado en la expendeduría más el coste en términos de mortalidad prematura. Dicho de otra manera, el placer de fumarse un paquete de cigarrillos -cuyo coste medio en 2010 se sitúa en los $3 €-$ vale para los fumadores al menos $107 €$ (hombres) y $75 €$ (mujeres).
Sin embargo, cualquier desviación del paradigma de la adicción racional implica la existencia de potencialmente sustanciales internalidades: daños sobre el propio consumidor no compensados por el placer del consumo. ¿Qué pasa con los fumadores que sólo valoran en $30 €$ el placer de fumar un paquete? Si fuman, es porque el coste en mortalidad es un coste diferido y, o bien no lo conocen, o bien no lo tienen en cuenta adecuadamente en su comportamiento presente. Un muy relevante ejemplo de comportamiento distinto al postulado por la teoría de la adicción racional es el que manifiestan los individuos con problemas de autocontrol -que derivan en preferencias temporalmente inconsistentes. En estos casos, los individuos conocen los costes totales de fumar y deciden que no fumar es la decisión que maximiza su bienestar, sin embargo no son capaces de realizar la acción deseada (abstenerse de comenzar a fumar o abandonar el hábito en caso de que ya sean fumadores). En estas circunstancias, los impuestos y las restricciones al consumo en lugares públicos 
son mecanismos que refuerzan el autocontrol $^{2-4}$. Dado el sustancial porcentaje de fumadores que desean abandonar el hábito, no parece que la incidencia de este tipo de fallo en la soberanía del consumidor sea marginal. Junto a nuestras estimaciones, ello hace pensar que las pérdidas de bienestar asociadas al consumo de tabaco son mucho más cuantiosas que lo que un cómputo de los costes externos podría sugerir. En este sentido las políticas de prevención y control del tabaquismo pueden generar considerables beneficios sociales.

\section{BIBLIOGRAFÍA}

1. Sloan FA, Ostermann J, Conover C, Taylor DH Jr. Picone G. The price of smoking. Cambridge: MIT Press; 2004.

2. Gruber J, Köszegi B. Is addiction rational? Theory and evidence. Q J Econ. 2001; 116(4): 1261-1303.

3. Gruber J, Köszegi B. Tax incidence when individuals are time-inconsistent: the case of cigarette excise taxes. J Public Econ. 2004; 88: 1959-1987.

4. Gruber J, Köszegi B. A Modern View of Tobacco Taxation. International Union Against Tuberculosis and Lung Disease. Paris; 2008.

5. Gruber J, Mullainathan S. Do cigarette taxes make smokers happier?. Advances in Econ. Anal.Pol. 2005; 5(1), Article 4.
6. Hersch J. Smoking restrictions as a self-control mechanism. J. Risk Uncertain. 2005; 31(1): 5-21.

7. Kan K. Cigarette smoking and self-control. J Health Econ. 2007; 26: 61-81.

8. Cutler DM. Health care and the public sector. En: Auerbach AJ, Feldstein M, Eds. Handbook of Public Economics. North Holland: Elsevier Science; 2000, p. 2145-2243.

9. Viscusi WK. The value of risks to life and health. J Econ Lit. 1993; 31(4): 1912-1946.

10. Viscusi WK, Hersch, J. The mortality costs to smokers. J Health Econ. 2008; 27: 943-958.

11. Albert C, Malo MA. Diferencias salariales y valoración de la vida humana en España. MC. 1995; 201: $87-125$.

12. Riera A, Ripoll AM, Sbert JM. Estimación del valor estadístico de la vida en España: una aplicación del método de los salarios hedónicos. Hacienda Pública Esp. 2007; 181(2): 29-48.

13. Martínez J, Méndez I. ¿Qué podemos saber sobre el Valor Estadístico de la Vida en España utilizando datos laborales?. Hacienda Pública Esp. 2009; 190: 73-93.

14. Panel de Hogares de la Unión Europea, Instituto Nacional de Estadística. http://www.ine.es/jaxi/ menu.do?type $=$ pcaxis $\&$ path $=/ \mathrm{t} 25 / \mathrm{p} 442 \&$ file $=$ inebase.

15. Encuesta de Accidentes de Trabajo, Ministerio de Trabajo e Inmigración. http://www.mtin.es/estadisticas/EAT/Welcome.htm. 


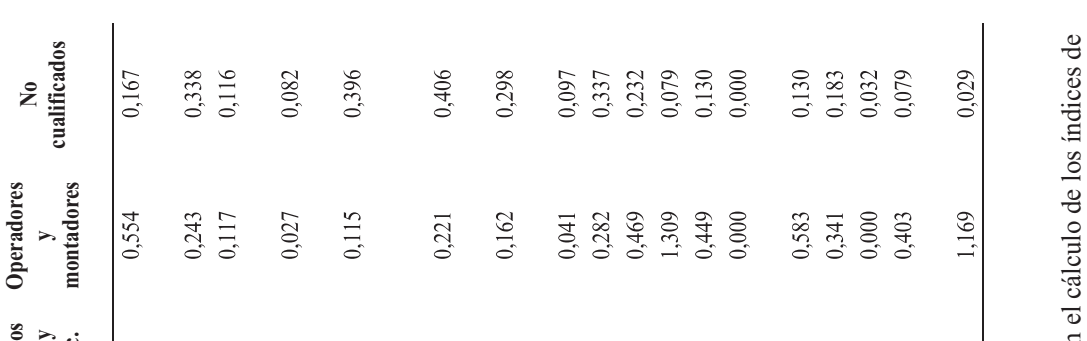

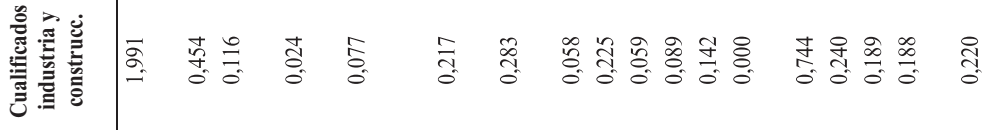

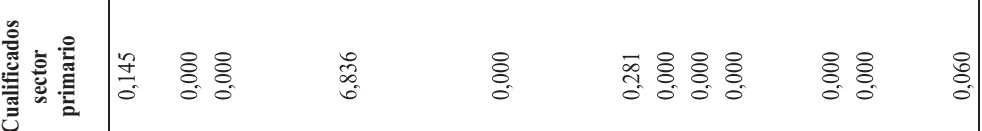

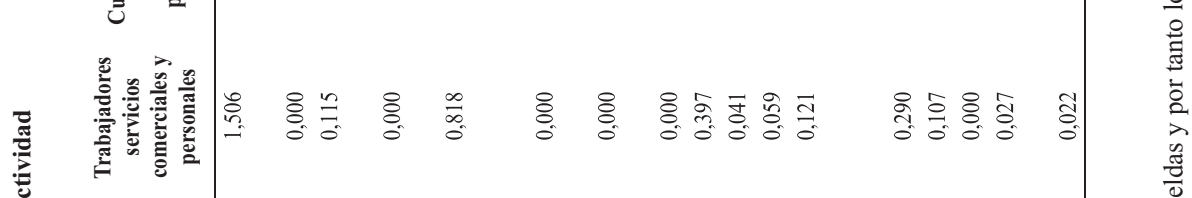

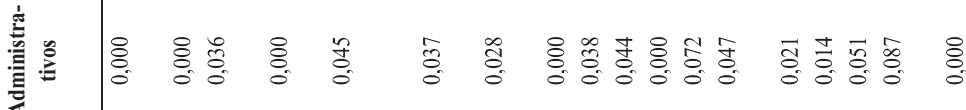

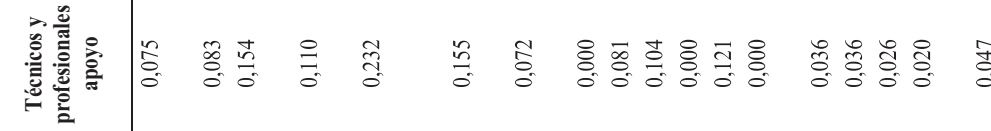

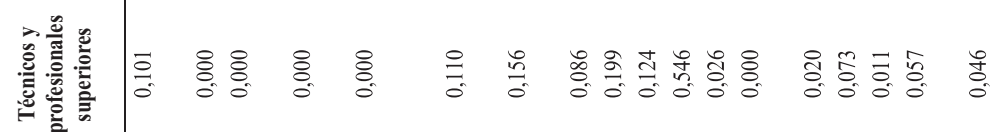

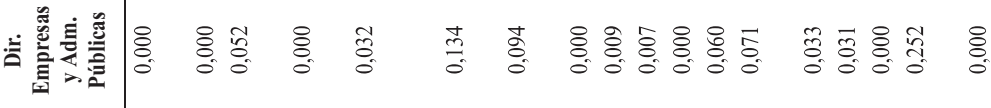

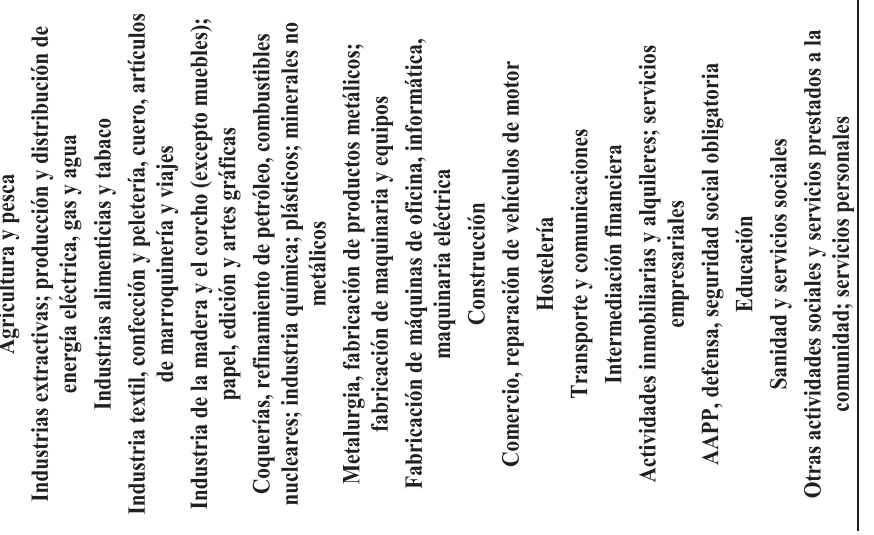

